\title{
Paychecks, Stress, and Variable-Free Semantics
}

\author{
Pauline Jacobson \\ Brown University \\ in Proceedings of the Tenth Conference on Semantics and Linguistic Theory, \\ Cornell: Cornell Working Papers in Linguistics, 2000.
}

\section{Introduction}

This paper examines the implications of contrastively stressed pronouns for two views of pronominal binding: the standard account which makes crucial use of variable names, and the variable-free account (see, among others, Jacobson, 1999, to appear). Although the existence of contrastive stress on bound pronouns appears at first glance to present a challenge to the variable-free approach and to instead require crucial reference to variable names, this challenge turns out to be illusory. In fact, I show here that once we broaden the landscape to include contrastively stressed paycheck pronouns, we find that the variable-free program makes exactly the right predictions in an interesting range of cases. The account of contrastive stress which relies on variable names, on the other hand, does not. This is not to say that there is no available account of the facts within the standard theory of pronominal binding - to consider all possible ways that the standard theory could handle contrastive stress on bound pronouns is, obviously, beyond the scope of this paper. Suffice it to say, though, that I will show that some of the more obvious available accounts of contrastive stress (combined with the standard account(s) of paycheck pronouns) will not account for the facts under consideration here. Interestingly, this difference between the two theories is not an accident tied to one particular implementation vs. another - the difference turns out to be a direct consequence of the two different approaches to binding.

First, let me briefly review the basic idea of, rationale for, and mechanics of the variable-free approach. The idea is that the semantic machinery makes no use of variables (and hence no use of assignment functions). The meaning of any expression is not - as in the standard account - a function from assignment functions to something else. Rather, the meaning of any expression which contains a pronoun which is unbound within that expression is a function from individuals to something else. (Note that I while I will in fact use variables in representing meanings, this is purely a notational device. One could in fact adopt a variable-free notation using combinators, but this is rather reader-unfriendly.)

There is more at stake here then just the question of how to treat pronominal binding. As discussed in some detail in Jacobson (1999, to appear), the variablefree program is motivated by the more general hypothesis of "direct compositionality". This hypothesis, familiar from Montague (1974), is that the syntax builds expressions while the semantics works in tandem to supply a modeltheoretic interpretation for each expression as it is built in the syntax. This is obviously an extremely elegant and simple conception of the syntax/semantics "interface". Among other advantages, it obviates the need for any intermediate level of LF and hence the need for any rules mapping surface forms into LFs. While I do not have space here to fully elucidate the connection between the hypothesis of direct compositionality and variable-free semantics, we can note that certain arguments for an abstract level of LF are crucially based on the assumption that "binding" is a relationship between two actual NPs (or, DPs), traces, and/or variables at some level of representation. In the variable-free account, "binding" is 
actually a relation between two argument slots rather than two actual linguistic elements - and so it turns out that many of the traditional arguments for abstract levels of representation such as LF evaporate once binding is recast in this way. Indeed, it is exactly this difference between the two approaches which will be crucial in the account of paycheck pronouns and contrastive stress, and so I return to this point in Sec. 7. In addition, this program simplifies the model-theoretic machinery by eliminating assignment functions, and it also has considerable additional empirical motivation documented in the papers referred to above.

A quick review of the basic mechanics is also in order. First, I assume that the meaning of a pronoun is the identity function on individuals (possibly with gender information built in) - hence of type $\langle\mathrm{e}, \mathrm{e}\rangle$. This will be refined below: the present paper explores the idea that these are identify functions over some contextually salient domain of individuals. Moreover, pronouns and/or expressions containing pronouns combine with other material by use of two type shift rules which regulate the combinatorics. The first is the "Geach rule" (Geach, 1972) which shifts a function $h$ of type $\langle a, b\rangle$ into a function of type $\langle<c, a\rangle,\langle c, b\rangle\rangle$ :

$$
\mathbf{g}(\mathrm{h})=\lambda \mathrm{V}[\lambda \mathrm{C}[\mathrm{h}(\mathrm{V}(\mathrm{C}))]] \quad(\text { for } \mathrm{V} \text { of type }<\mathrm{c}, \mathrm{a}>\text { and } \mathrm{C} \text { of type } \mathrm{c})
$$

The intuitive idea is perhaps simplest to grasp by noting that $\mathbf{g}$ is a unary ("Curry'ed") version of function composition; thus $\mathbf{g}(\mathrm{h})(\mathrm{f})=\mathrm{h}$ o $\mathrm{f}$. Note that pronouns are of type <e,e> (while ordinary NPs are of type e), and material containing pronouns which are unbound within that material are of type $\langle e, a\rangle$, for some a (while corresponding expressions which contain no unbound pronouns within them are of type a). Thus the $\mathbf{g}$ rule is what allows a function which wants an argument of type e - or, more generally, of some type a - to shift so that it can instead take as argument a pronoun (of type $\langle\mathrm{e}, \mathrm{e}\rangle$ ) or, more generally, a pronoun containing constituent (of type $\langle\mathrm{e}, \mathrm{a}\rangle$ ). To illustrate, take the derivation of he lost. lost' is of type <e,t> but it can undergo $\mathbf{g}$ to allow its argument to be of type <e,e> (in this case, the identity function on individual), as follows:

$$
\begin{aligned}
& \text { lost' <e,t> ---> g(lost') }=\lambda \mathrm{f}\left[\lambda \mathrm{x}\left[\operatorname{lost}^{\prime}(\mathrm{f}(\mathrm{x}))\right]\right](\text { for } \mathrm{f} \text { of type }<\mathrm{e}, \mathrm{e}>) \\
& \text { he lost; }=\mathbf{g}\left(\text { lost }^{\prime}\right)\left(\mathrm{he}^{\prime}\right)=\lambda \mathrm{f}\left[\lambda \mathrm{x}\left[\operatorname{lost}^{\prime}(\mathrm{f}(\mathrm{x}))\right]\right](\lambda \mathrm{y}[\mathrm{y}])=\operatorname{lost}^{\prime}
\end{aligned}
$$

Similarly, his mother is of type <e,e> (and denotes the-mother-of' function). Hence his mother lost is composed as shown in (3):

$$
\begin{aligned}
\mathbf{g}\left(\text { lost' }^{\prime}\right)(\text { the-mother-of' }) & =\lambda \mathrm{f}\left[\lambda x\left[\operatorname{lost}^{\prime}(\mathrm{f}(\mathrm{x}))\right]\right](\text { the}- \text { mother-of' } \\
& =\lambda \mathrm{x}\left[\operatorname{lost}^{\prime}\left(\text { the }^{\prime} \text {-mother-of' }(\mathrm{x})\right)\right]
\end{aligned}
$$

The above illustrates cases where an argument of a function contains an unbound pronoun, and where the pronoun remains unbound after the two combine. But of course pronouns can also eventually be bound, and this is accomplished by a second type-shift rule which I have dubbed $\mathbf{z}$. This takes a function $h$ of type $\langle\mathrm{a},\langle\mathrm{e}, \mathrm{b}\rangle\rangle$ and shifts it to a function of type $\langle\langle\mathrm{e}, \mathrm{a}\rangle,\langle\mathrm{e}, \mathrm{b}\rangle\rangle$ in such a way as to "merge" the two e-argument slots. This is spelled out formally in (4):

$$
\mathbf{z}(\mathrm{h})=\lambda \mathrm{f}[\lambda \mathrm{x}[\mathrm{h}(\mathrm{f}(\mathrm{x}))(\mathrm{x})]] \quad(\text { for } \mathrm{f} \text { of type }<\mathrm{e}, \mathrm{a}>)
$$

To elucidate this informally, consider love' which is a relation between individuals. $\underline{\mathbf{z}(\text { love') }}$ is thus a relation between individuals and functions of type 
$<\mathrm{e}, \mathrm{e}>$ such that to $\mathbf{z}($ love') some function $\mathrm{f}$ is to be an $\mathrm{x}$ who loves $\mathrm{f}(\mathrm{x})$. With this much apparatus, here is the derivation of Every man loves his $_{i}$ mother:

$$
\begin{aligned}
& \text { loves' ---> } \mathbf{z}(\text { loves' })=\lambda \mathrm{f}[\lambda \mathrm{x}[\text { loves' }(\mathrm{f}(\mathrm{x}))(\mathrm{x})]] \\
& \text { loves-his-mother' }=\mathbf{z}\left(\text { loves }^{\prime}\right)(\text { his-mother' })=\lambda f\left[\lambda x\left[\operatorname{loves}^{\prime}(\mathrm{f}(\mathrm{x}))(\mathrm{x})\right]\right](\text { the- } \\
& \text { mother-of') }=\lambda x \text { [loves'(the-mother-of' }(x))(x)] \\
& \text { every man loves his mother: every-man' }\left(\lambda x \left[\text { loves' }^{\prime}(\text { the-mother-of'(x))(x)]) }\right.\right.
\end{aligned}
$$

Finally, we need to say a word about free pronouns, as in (6):

His mother lost.

The idea here is that this is simply a function from individuals to propositions (thus it is the function $\underline{\lambda x}$ [lost'(the-mother-of'(x))]) rather than a proposition. However, in order to extract propositional information from this (as, for example, to compute inferences), the listener applies this function to some contextually salient individual. Note that in the standard theory (6) is also not proposition - rather, it is a function from assignment functions to propositions. Moreover, its value varies according to which assignment function is chosen (unlike the case of fully closed expressions, which are constant functions from assignment functions). Thus here too we cannot arrive at propositional information unless we posit that this is applied to something else - in this case, to a contextually salient assignment function.

\section{An apparent challenge}

A central claim which follows from this program (or, which can be seen as a motivation for the program) is the following: whenever there are two pronouns which - in the standard account - have different variable names but find themselves in formulas which are alphabetic variants of each other, the two pronouns always make the same semantic contribution. Put more informally, the claim here is that a difference in variable names should never matter: variable names themselves are just meaningless artefacts.

But there is an intriguing challenge to this claim which is developed in Sauerland (1998, 1999). Thus Sauerland argues that the distribution of contrastive stress on bound pronouns provides evidence that different variable names are in fact semantically different objects. So, for example, consider (7): ${ }^{1}$

Every third grade boy ${ }_{i}$ loves his ${ }_{i}$ mother, while every FOURTH grade boyj $_{j}$ HATES hisj/ HIS j $_{j}$ mother.

I will not assume any particular theory of contrastive stress in this paper, but it is clear that all of the stressed items in cases like (7) must contrast with something else. (In this and all of the subsequent examples involving contrastive stress, the corresponding items in the first clause can also be stressed, although generally this is not required. I will systematically suppress the stress in the first clause.) The obvious question raised by (7) is: just what is the stressed pronoun contrasting with? (We return momentarily to the version of (7) in which the pronoun is not stressed.) In the variable-free semantics as developed in, e.g., Jacobson (1991, 1999) (see also the version in Hepple, 1990), both pronouns denote the identity function. Since they have the same meaning, there should be no contrast. (Note 
that there are other versions of variable-free semantics; thus both Szabolcsi (1992) and Dowty 1999) explore versions in which the pronouns have "fancier" meanings such that binding is built in to the pronoun meanings. But the same point holds; in those accounts too the two pronouns in (7) have the same meaning.) As Sauerland notes, however, there appears to be no mystery if we take the standard semantics making use of variable names. Since the two pronouns in (7) can have different variable names they can contrast; thus the meaning (and/or LF) of (7) can be represented roughly as in (8):

every third grade boy $(\lambda x[x$ loves $x$ 's mother $])$ while

every fourth grade boy $(\lambda y[y$ hates $y$ 's mother $])$

Notice, incidentally, that contrastive stress is not required (as shown in (7)). The most straightforward account of this in the standard semantics is to note that in the usual way to set things up the two pronouns can indeed be the same variable. Thus the second conjunct in (8) could also have as its representation every fourth grade boy ( $\lambda x[\mathrm{x}$ hates $\mathrm{x}$ 's mother $]$ ). (although it should be noted that Sauerland's actual solution is somewhat different here).

Before continuing, let me make an interesting side observation which will prove useful below. Consider a sentence like (9):

$$
\text { Every fourth grade boy } \mathrm{i}_{\mathrm{i}} \text { hates HIS } \mathrm{S}_{\mathrm{i}} \text { mother. }
$$

Contrastive stress here is felicitous in two different circumstances. The first is the one that we see in (7), where the bound pronouns is being contrasted with some other bound pronoun. The second is one where the contrast is with some other individual(s) in the discourse context (HIS, rather than, say John's mother). Interestingly, stress on reflexive pronouns disambiguates these two uses of contrastive stress. In the case where the contrast is with others in the discourse context, the stress falls on SELF (as in (10)); in the case where the contrast is with another bound pronoun the stress falls on HIM (as in (11)):

(10) Every third grade boy loves Mary, and every FOURTH grade boy loves himSELF/*HIMself (as opposed to someone else).

(11) Every third grade boy loves himself, and every FOURTH grade boy loves HIMself/*himSELF (*as opposed to someone else).

\section{An initial problem for the "different variable names" solution}

Returning to the main theme, we can see that a closer look immediately reveals that the "different variable names" solution is, in fact, quite suspect: the places where contrastive stress is allowed does not line up with the situations which (under standard understanding) involve a difference in variable names. We begin with an observation that Sauerland himself notes and attributes to Heim (personal communication); this is the fact that contrastive stress is not very good in (12):

(12) a. I told every student to call his mother, but only every YOUNG student called his/*HIS mother. (Sauerland, 1998, attributed to Heim, p.c.)

b. I told every boy to wash himself, but only every FOURTH grade boy washed himself/*HIMself. 
Thus, as Sauerland notes, contrastive stress is not possible if one of the binders quantifies over a domain which is a subset of the domain quantified over by the other binder. Actually, there is an irrelevant complication surrounding (12) to which I return below, so let us instead explore the point using a simpler case. Thus note that contrastive stress is clearly impossible in (13)-(14) where the two binders quantify over exactly the same domains:

(13) Every third grade boy ${ }_{i}$ loves his $s_{i}$ mother, but no third grade boy ${ }_{j}$ CALLED his $/$ * HIS $\mathrm{j}_{\mathrm{j}}$ mother.

(14) Every third grade boy ${ }_{i}$ washed himself $f_{i}$, but no third grade boy $y_{j}$ SHAVED himself $\mathrm{j} / *$ HIMself $_{\mathrm{j}}$.

Thus note that (14) compares quite robustly with (15):

(15) Every third grade boy $\mathrm{i}_{\mathrm{i}}$ washed himself $\mathrm{f}_{\mathrm{i}}$, and no/every FOURTH grade boy S SHAVED himself $_{\mathrm{j}} / \mathrm{HIMself}_{\mathrm{j}}$.

Obviously, these facts cast serious doubt on the "different variable names" solution. After all, nothing in the usual understanding of variables precludes a representation for, e.g., (13) in which the two pronouns also correspond to different variables, as they are indeed bound by different material (it just happens to be material quantifying over the same domains):

$$
\begin{array}{r}
(=\text { representation for }(13)) \text { : every third grade boy } \lambda x[x \text { loves x's mother }] \\
\text { but no third grade boy } \lambda y[y \text { called y's mother }]
\end{array}
$$

In fact, the actual generalization appears to be the following: The pronouns can contrast just in case the domains of quantification of the two binders are in contrast. There are a couple of points to note here. First, one might think that this generalization is not quite right in that $Y O U N G$ can be stressed in (12a):

(12a) I told every student to call his mother, but only every YOUNG student called his/*HIS mother.

Nonetheless, I believe that the stress on $Y O U N G$ here is not due to a contrast with every student in the first conjunct but rather that YOUNG is contrasting with some implicit property, e.g., "old". This in turn raises the question of why we can't stress HIS where the contrast set is also implicit (i.e., where there is an implicit contrasting proposition no old student called his mother). Indeed, I believe that this is marginally possible (despite my use of the asterisk above), especially if the sentence ends with a rising intonation suggesting an implicit continuation. Nonetheless, it is much easier to get stress on YOUNG in virtue of the implicit contrast than on HIS, and I leave it open as to why this is so.

Second, the fact that stress is possible on the pronoun just in case it is possible on the binder might lead one to believe that we are dealing with a kind of agreement phenomenon here. If this were the case then the account of this in the variable-free program would be quite trivial. ${ }^{2}$ However, I do not believe that this view can be maintained. For one thing, it is not obvious how this would account for the two different stress patterns on reflexive pronouns. Moreover, consider a case like (17) where stress on the pronoun is impossible:

(17) Every third grade boy ran, and every FOURTH grade boy DANCED with his MOTHER/*HIS mother/*HIS MOTHER. 
Stress on MOTHER here is presumably a consequence of the fact that the entire second VP receives contrastive stress (I believe that stress on DANCE is in fact optional here). The important point, though, is that stress on HIS is impossible presumably because it is not in contrast with any other bound pronoun. If this were a simple matter of agreement, then the stress on FOURTH grade boy (i.e., on the "binder") should be sufficient to license stress on the bound pronoun.

\section{A solution in variable-free semantics}

As we see above, the facts in (13) and (14) cast severe doubt on the "different variable names" explanation for the possibility of contrastive stress on bound pronouns. The remainder of this paper will elucidate further problems with this explanation. But now let us reconsider the variable-free approach, and note that there is an obvious tack that one might try (indeed, this is mentioned although not pursued in unpublished work by Sauerland). This is to allow the two pronouns to be the identity function over different domains, and this of course would explain their contrast. There are some interesting open questions as to just how to think of this: clearly we do not want to think of this as part of the lexical meaning since we of course do not want to say that there are in the lexicon an infinite number of accidentally homophonous pronouns. Rather, let us assume that pronouns denote the identity function over individuals in some contextually salient domain, and that if the context supplies different domains for each of the pronouns then they are in contrast (even though, strictly speaking, their meanings are the same). Consider, then, the original sentence in (7). The idea is that the first his can be understood as the identity function over the domain of third grade boys, while the second his is understood as the identity function over the domain of fourth grade boys.

Note that the above generalization concerning contrastive stress follows: the pronouns can contrast just in case the domain of quantification of their "binders" are in contrast. I have put "binders" here in quotes because this terminology is somewhat misleading in the variable-free account - the binding is actually the semantic merging of two argument slots via the $\mathbf{z}$ rule rather than involving any kind of co-indexation or other relationship between the "binder" and the pronoun. I assume, however, that it will cause no confusion to continue to use the term "binder" in this way. Note further that - despite the fact that there is no actual coindexation or any other kind of syntactic relationship between the "binder" and the pronoun - the generalization follows. If the two pronouns are identity functions over contrasting domains then the two VPs will also be functions from different (and contrasting) domains. Hence it would be impossible to apply the two VPs to generalized quantifiers built on the same domain. Finally, note that it also follows immediately that contrastive stress is not required in (7), since the two pronouns can both be understood as the identity function over broader domains (e.g., the domain of all individuals, of all boys, etc.)

The strategy for the remainder of this paper is to explore the distribution of contrastive stress on paycheck pronouns, and to show that things work out exactly right in the variable-free account but do not work out under the "different variable

names" solution. I will moreover show that this is due to the following difference between the two views on binding. The standard view of binding requires an actual linguistic expression (a variable) in the "bound" position, and binding is generally accomplished by $\lambda$-abstraction over this variable. In the variable-free view, binding is a merging of argument slots (by the $\mathbf{z}$ rule). As we will see, this difference has a significant consequence for the analysis of paycheck pronouns, 
which in turn impacts on the account of when these can be contrastively stressed. But before looking at the paycheck situation, an interlude.

\section{Interlude: Some initial pleasing consequences}

There are two pleasing consequences to the above suggestion. The first centers on the two different stress patterns for reflexive pronouns. Assume that an ordinary stressed NP invokes a set of alternative individuals (as in, e.g., Rooth, 1985). What would contrastive stress on a pronoun mean? In the variable-free view, there are two obvious ways to think about alternatives to pronoun meanings. One is that a stressed pronoun invokes a function from individuals to a set of alternative individuals. A second is that it invokes a set of alternative functions - in this case a set of identity functions over alternative domains. (It may be that there are cases where other alternative functions of type $\langle\mathrm{e}, \mathrm{e}\rangle$ are invoked.) The two different stress patterns on reflexives appear to reflect just these two possibilities: [[himSELF]] is the first case where what is invoked is a function from individuals to a set of alternative individuals (rather than to self), while [[HIMself]] invokes a set of identity functions over different domains. (I leave it as an open question as to just exactly why the particular stress patterns correlate with each possibility. )

A second consequence centers on VP Ellipsis. Let us assume that in the case of an elliptical VP, the "missing" VP meaning generally likes to be the meaning of some other overt VP. (Note that the received position here is that the missing VP meaning must be the meaning of some other overt VP - this follows either under deletion approaches to VP Ellipsis or under the standard interpretive approaches whereby ellipsis involves picking up the meaning or copying the LF of the antecedent VP. I actually assume that this is incorrect and that VP "Ellipsis" is a case of "deep anaphora", where there is a missing complement to an auxiliary and where the missing property meaning is contextually supplied. However, I assume further that properties of the type which need to be supplied in VP ellipsis are very "fragile" and difficult to access without heavy contextual support - hence these are most accessible if they are made salient by being the meaning of some actual overt VP. In the end, then, this view entails that VP ellipsis remains a valid diagnostic for the meaning of the antecedent VP. Note that the assumption that the antecedent property can on occasion be contextually supplied is not crucial ; the same points will go through under the "received" position.) Under the variable-free view, the existence of a sloppy reading in (18) is due to the fact that the meaning of the

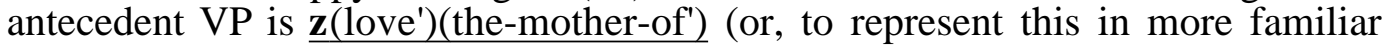

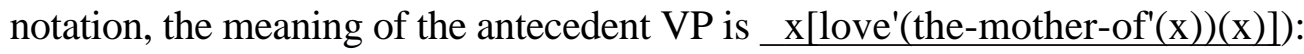

$$
\text { Every } 3 d \text { grade boy } \text { loves his }_{\mathrm{i}} \text { mother. Every fourth grade boy does too. }
$$

Now consider (19):

(19) Every 3d grade boy ${ }_{i}$ loves his mother. Every FOURTH grade boy ${ }_{j}$ HATES HIS ${ }_{\mathrm{j}}$ mother; and ??every FIFTH grade boy $\mathrm{k}_{\mathrm{k}}$ does too

It is difficult to get the meaning where the missing VP is understood as hates hisk mother. This follows from the account developed so far; if HIS in the second conjunct is stressed it must be that it is understood as the identity function over 4th grade boys while his in the first conjunct is understood as the identity function over $3 \mathrm{~d}$ grade boys. Hence the entire VP meaning of the 4th-grade clause is a function from the domain of fourth grade boys to propositions, and thus is inappropriate to be supplied as the missing material (and yield the sloppy reading) in (19). Note 
that the sloppy reading for (19) is not completely impossible. But then again, we would not really expect it to be: it should be more or less on a par with the availability of a sloppy reading in (20):

(20) ??John scratched his arm, and Mary did too.

As noted in Ross (1967), some speakers have a problem with sloppy identity in the case that there is a gender mismatch. This is not surprising if gender is (at least in part) semantic - the first conjunct supplies the self-arm-scratching property defined for male individuals. However, (20) is not completely impossible for most speakers, and hence the marginal availability of the sloppy reading in (19) is also unsurprising.

\section{Stress and paycheck pronouns: the puzzle for the different variable names solution}

\subsection{Paycheck pronouns in a theory with variables}

Consider a typical paycheck pronoun, as in (21):

(21) Every $3 \mathrm{~d}$ grade boy loves his mother; every FOURTH grade boy HATES her.

There are two major accounts within the standard theory. The first is the "free function variable theory"; this is proposed in Engdahl (1986) and is a modification of the proposal in Cooper (1979). According to this, her has the complex meaning $\mathrm{f}(\mathrm{x})$, where $\mathrm{f}$ is a variable over functions of type $\langle\mathrm{e}, \mathrm{e}\rangle$. It remains free, and picks up the contextually salient mother-of function, while $\mathrm{x}$ is bound by the subject in the normal way that variable-binding takes place. The second account is the "pronouns of laziness" approach proposed originally in Karttunen (1969) and further argued for in Partee (1975), Jacobson (1977) and revived in more recent work such as Heim (1990) (although the latter, strictly speaking, is concerned with donkey rather than paycheck pronouns). Under this view her in (21) has as its representation the full NP the mother of $\mathrm{x}$. Thus it is represented as a full NP with a variable within it which is bound in the normal way.

Since I will ultimately be adopting a variant of the Cooper/Engdahl account, let me give here two arguments for this type of solution as opposed to the pronouns of laziness analysis. First, the former predicts that a paycheck pronoun is a "deep anaphor". That is, since the value of the free variable $f$ is contextually supplied, it should not require overt linguistic material as its antecedent. And indeed this prediction is correct - consider the following scenario:

(22) A new faculty member is in the mail room, picking up her first paycheck. Waving it in the air, she says: What am I supposed to do with this? Other faculty member answers: Well, most of us used to put it in the Brown Employees' Credit Union; now we've all learned to put it in the bank.

The paycheck reading for $i t$ seems quite effortless. ${ }^{3}$ Second, notice that in contrast to (20), there is no gender clash in (23) - the paycheck reading is perfect:

(23) Every 3d grade boy loves his mother. Every 3d grade GIRL HATES her. 
This follows under the Cooper/Engdahl approach since the value of $\mathrm{f}$ can be supplied by the meaning of mother, and hence there will be no gender clash. The VP Ellipsis situation in (20) is rather different, for here the phrase whose meaning makes salient the relevant property must be the full VP scratch his arm - and this has gender information built in. Under the pronouns of laziness solution the ability to have the gender mismatch in (23) is surprising - there ought to be at least some cost to having the antecedent NP be different from the representation of the paycheck pronoun. (Of course, we would not expect a problem if what is required is identity of LFs and if it is the case that gender is entirely syntactic and is not represented at LF. But then it is not clear what accounts for the oddness of the VP Ellipsis case.) There are, then, reasons to reject the pronouns of laziness approach. But to be on the safe side, I will consider the implications of the data below for both the Cooper/Engdahl view and for the pronouns of laziness analysis.

Before moving on, though, let me note that there is one aspect of both accounts of paycheck pronouns which seems unsatisfactory. This is that under either view, paycheck pronouns are just accidentally homophonous with ordinary pronouns. (Strictly speaking, this is not true in Engdahl's analysis, but her way of collapsing ordinary pronoun meanings with the paycheck meanings relies on what is arguably a terminological trick, which is to invoke the notion of a "0-place function". See Jacobson, to appear, for more discussion.) While I of course cannot prove that this is not a case of accidental homophony it seems quite unlikely that it is (especially in view of the fact that the full set of pronouns must be each be homophonous in this way). We return to this below, to show that this problem disappears in the variable-free view: the existence of paycheck readings follows from the general apparatus used for binding.

\subsection{The puzzle: the distribution of stress on paycheck pronouns}

We are now in a position to consider the central observation in this paper. This is a two-prong observation, the first prong of which is the impossibility of contrastive stress on the paycheck pronoun in (24). Thus compare (24) - where we have a paycheck pronoun - with (25) - where we have the corresponding full NP and where stress on the pronoun (variable) part of that NP is just fine:

*Every 3d grade boy loves his mother, while every 4th grade boy HATES HER.

Every $3 \mathrm{~d}$ grade boy loves his mother, while every 4 th grade boy HATES HIS mother.

The problem here is quite obvious: whether we take the free function variable approach or the pronouns of laziness approach, the paycheck pronoun contains a variable whose name differs from the corresponding variable in the NP in the first conjunct. To elucidate, consider first the free function variable account; under this account the representation for (24) is (26):

$$
\begin{aligned}
& \text { every-3d-grade-boy' }(\lambda x[x \text { loves the-mother-of' }(x)]) \quad \text { while } \\
& \text { every-4th-grade-by' }(\lambda y[y \text { hates } \underline{\mathrm{f}(\mathrm{y})]})
\end{aligned}
$$

Notice that we should have two points of contrast here if we think purely in terms of the representations: $\underline{x}$ contrasts with $\underline{y}$ and $\underline{f}$ contrasts with the-mother-of'. One might easily argue that the second is not a true contrast since $f$ is ultimately "anaphoric" to the-mother-of' (thus it is a variable which remains free and whose 
value is ultimately fixed by the context but is fixed as the meaning of the-mother$\underline{\text { of'}})$. Nonetheless, we still have a contrast between $\underline{x}$ and $y$.

Exactly parallel remarks hold under the pronouns of laziness approach. Here the representation for (24) is (27):

$$
\begin{aligned}
& \text { every-3d-grade-boy' }(\lambda x[x \text { loves the-mother-of' }(x)]) \quad \text { while } \\
& \text { every-4th-grade-by' }(\lambda y[y \text { hates the-mother-of' }(y)])
\end{aligned}
$$

Once again there should be a contrast, since we have the two different variable names $\underline{x}$ and $\underline{y}$ as part of each meaning. In other words, we would expect stress to be allowed exactly as it is in (25).

Now at this point there is an obvious solution available; we can conjecture that the domain here is simply too big - the paycheck pronoun is not the minimally contrasting domain (thus only the variables contrast, and not the rest of the material surrounding the variables). (Depending on just how one works things out, this could fall out from, e.g., the account of stress in Schwarzschild (1999).) Or, one might refine this to say that contrastive stress on the paycheck pronoun is possible only if the head part of its complex representation is in contrast with something else (notice that under the Cooper/Engdahl approach this would require us to think of the function variable as the head). ${ }^{4}$

But this type of solution will not do. To see this, we now move to the second prong of the observations here. This is that contrastive stress on the paycheck pronoun in (28) is perfect:

(28) Every $\operatorname{man}_{i}$ who loves his $s_{i}$ mother thinks that she $e_{f(i)}$ is nice, while every man $_{\mathrm{j}}$ who HATES HIS $\mathrm{j}_{\mathrm{j}}$ mother thinks that $\mathrm{SHE}_{\mathrm{f}(\mathrm{j})}$ is a jerk.

The key point about this example is that here too the individual variables within the complex LF of the two paycheck pronouns can be different variables. On the other hand, the rest of the material surrounding these individual variables is identical in both. So whatever principle is invoked to rule out contrastive stress on the paycheck pronoun in (24) should rule it out here as well.

To clarify, compare the representations of (24) vs. (28) under either of the approaches to paycheck pronouns; (29) and (30) shows these under the Cooper/Engdahl approach while (31) and (32) shows this under the pronouns of laziness approach:

$$
\begin{aligned}
& \text { every-3d-grade-boy' }(\lambda x[x \text { loves the-mother-of' }(x)]) \quad \text { while } \\
& \text { every-4th-grade-by' }(\lambda \mathrm{y}[\mathrm{y} \text { hates } \underline{\mathrm{f}(\mathrm{y})}]) \quad \text { contrastive stress not allowed } \\
& \operatorname{every}^{\prime}\left(\lambda x\left[\operatorname{man}^{\prime}(\mathrm{x}) \& \text { love'}^{\prime}\left(\text { the-mother-of' }^{\prime}(\mathrm{x})\right)(\mathrm{x})\right]\right) \quad\left(\lambda \mathrm{x}\left[\operatorname{think}^{\prime}\left(\operatorname{nice}^{\prime}(\underline{\mathrm{f}(\mathrm{x})})\right]\right)\right. \\
& \operatorname{every}^{\prime}\left(\lambda y \left[\operatorname { m a n } ^ { \prime } ( \mathrm { y } ) \& \text { hate'(the-mother-of'(y))(y)]) } \left(\lambda y\left[\operatorname{think}^{\prime}\left(\operatorname{jerk}^{\prime}(\underline{\mathrm{f}(\mathrm{y})})\right]\right)\right.\right.\right. \\
& \text { or - second paycheck pronoun might be } \mathrm{g}(\mathrm{y}) \\
& \text { contrastive stress allowed } \\
& \text { every-3d-grade-boy' }(\lambda x[x \text { loves the-mother-of'(x)] }) \quad \text { while } \\
& \text { every-4th-grade-by' }(\lambda y[y \text { hates the-mother-of' }(y)]) \\
& \text { contrastive stress not allowed }
\end{aligned}
$$




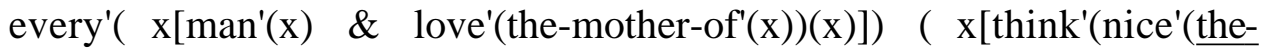
mother-of'(x))]) while

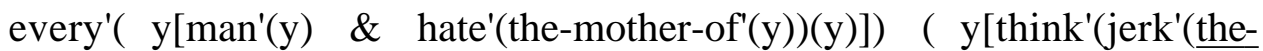
mother-of'(y))]) contrastive stress allowed

\section{The solution in a variable-free semantics}

\subsection{Paycheck pronouns in a variable-free semantics}

We turn now to the analysis of paycheck pronouns in a variable-free semantics, to show that the above contrast falls into place. By way of background, we will first sketch the approach to paycheck pronouns developed in detail in Jacobson (to appear; see also Jacobson, 1991). This approach takes as its point of departure the basic idea of the Cooper/Engdahl approach, but modifies it to be compatible with the variable-free program. We will introduce the modification in two steps:

Step 1: Let the paycheck reading of her in (21) be simply a free function variable - rather than, as in Cooper/Engdahl, a complex meaning $\mathrm{f}(\mathrm{x})$. That is, in this step we eliminate the use of an individual variable as argument of the function. The key point to note is that under the variable-free account of binding, there is no need to supply the individual variable here. Rather, the argument slot of $\underline{f}$ is bound by application of the $\mathbf{z}$ rule applies to hates:

$$
\begin{aligned}
& \text { every 4th-grade-boy }(\mathbf{z} \text {-hates } f)= \\
& \text { every 4th-grade-boy }\left(\lambda \mathrm{g}\left[\lambda \mathrm{x}\left[\text { hates }^{\prime}(\mathrm{g}(\mathrm{x}))(\mathrm{x})\right]\right](\mathrm{f})\right) \text { - } \\
& \text { every 4th-grade boy' }\left(\lambda \mathrm{x}\left[\text { hates' }^{\prime}(\mathrm{f}(\mathrm{x}))(\mathrm{x})\right]\right.
\end{aligned}
$$

However there are two problems with the account so far. First, while we have eliminated the individual argument variable, we are still using a free variable over functions - obviously this is illegitimate in a variable-free semantics as it makes crucial use of variables. Second, there is still accidental homophony between a paycheck pronoun and a regular pronoun.

Step 2: Both problems can be solved at once. As to the illegitimate use of the free variable $\mathrm{f}$, the obvious solution is to treat her not as a free variable but rather as the identity function over functions of type <e,e>. The combinatorics now work in a fashion which is exactly analogous to what happens for the case of an ordinary unbound pronoun. To illustrate, take Every 4th grade boy hates her on the reading where her is ultimately understood as some contextually salient individual. (In other words, take this on the "ordinary" and not the paycheck reading). This is composed by applying $\mathbf{g}$ on hates and on every 4th grade boy; the final result of the whole sentence is $\lambda x[$ every-fourth-grade-boy'(hates'(x))]. As noted earlier, the "free" reading of a pronoun is one where the entire sentence does not denote a proposition but rather a function of type $\langle e, t\rangle$, and it is ultimately applied to some contextually salient individual.

Now consider this on the paycheck reading. Here we end up with a meaning of type $\langle<e, e\rangle, t>$ and so here this is applied to some contextually salient function. The composition of this meaning is similar to the case where she is understood as an ordinary free pronoun, but there are two differences. One is that the application of $g$ at each point introduces a new argument slot of type <e,e $>$ rather than a new argument slot of type e. Moreover, hates undergoes $\mathbf{z}$ (as well as $\mathbf{g}$ ) and this is what allows its subject to bind the argument position of the $\langle\mathrm{e}, \mathrm{e}\rangle$ 
function. Space precludes showing the full details, but the interested reader can verify that the derivation shown in combinatory form in (34a) will indeed yield the meaning shown in (34b):

a. $\mathbf{g}($ every-4th-grade-boy" $)\left(\mathbf{g}\left(\mathbf{z}\left(\right.\right.\right.$ hates' $\left.\left.^{\prime}\right)\right)\left(\right.$ her' $\left.^{\prime}\right)\left(\right.$ where $\left.h e r^{\prime}=\lambda \mathrm{f}[\mathrm{f}]\right)$

b. $\lambda f\left[\right.$ every-4th-grade-boy') $\left(\lambda x\left[\right.\right.$ hates' $\left.\left.^{\prime}(\mathrm{f}(\mathrm{x}))(\mathrm{x})\right]\right)$

Consider now the other remaining problem: we want to avoid accidental homophony between the paycheck reading and the ordinary reading of a pronoun. But, as pointed out to me by Mark Hepple, the paycheck reading actually comes for free from the $\mathbf{g}$ rule. Thus the fact that a pronoun can be understood as the identity function over ordinary individuals or as the identity function over functions of type $\langle\mathrm{e}, \mathrm{e}\rangle$ is automatic. To see this, note that the paycheck meaning of her is derived from its ordinary meaning by $\mathbf{g}$ as shown in (35):

$$
\text { her'; } \lambda x[x] ~--->g(h e r ')=\lambda f[\lambda y[\lambda x[x](f(y))]]=\lambda f[\lambda y[f(y)]]=\lambda f[f]
$$

Moreover, as noted in fn. 4, there are actually an infinite number of paycheck pronouns But it turns out that all of the more complex paycheck meanings just involve further applications of $\mathbf{g}$ (see Jacobson, to appear for details).

\subsection{Accounting for the Stress Puzzle}

We are now in a position to account for the stress puzzle. Consider again (36), where contrastive stress on the paycheck pronoun is impossible:

\section{Every $3 \mathrm{~d}$ grade boy loves his mother. Every 4th grade boy HATES} her/*HER

Recall that the problem for the "different variable names" solution is that his mother and her can contain individual variables with different names. This is a consequence of the fact that the paycheck pronoun her has a complex representation/meaning. But, one might ask the following: Could an analogous problem arise in the variable-free account? In fact it does not, but to see why not we will go down a garden path in which it appears that the same problem does rear its head under variable-free. Thus, we have said that an ordinary pronoun is the identity functions over contextually salient domain of individuals. A paycheck pronoun is the identity function over functions of type $\langle\mathrm{e}, \mathrm{e}\rangle$. It is perfectly plausible, then, to suppose that a paycheck pronoun also allows for a domain restriction; thus assume that a paycheck pronoun is the identity function over some contextually salient domain of functions of type $\langle\mathrm{e}, \mathrm{e}\rangle$. But now we appear to be back to the initial problem. Suppose that his in the first conjunct is understood as the identity function over the domain of $3 \mathrm{~d}$ grade boys. Then his mother is the mother-of function defined for the domain of $3 \mathrm{~d}$ grade boys. Now let the paycheck pronoun be the identity function over functions with domain 4th grade boys. The her does contrast with his mother, and so contrastive stress should be allowed.

Fortunately, the tale spun above neglected one important fact - and this is that the paycheck pronoun her is anaphoric to his mother (or, to mother.) This is actually a somewhat oversimplified way to put it: the more accurate way to phrase this is to say that every 4th grade boy hates her has the meaning represented earlier in $(34 b)$ - it is thus looking for a contextually salient function to be applied to. Suppose, then, her was stressed in virtue of the fact that it is understood as the identity function over functions with domain 4th grade boys. (The contrast, then, 
is with his mother in the first clause, which is a function with domain third grade boys.) Then every 4th grade boy hates her is "looking" for some contextually salient function whose domain is 4th grade boys. Obviously, then, this cannot be supplied by the meaning of his mother in the first clause. That NP would be understood as the mother-of function defined for the domain of $3 \mathrm{~d}$ grade boys. Or, if his is understood broadly, then it is the full mother-of function (i.e., with the full domain), but this still could not supply the necessary function which (34) is looking for. Note that the same remarks hold for the meaning of mother - this could not supply the contextually relevant function for the same reason.

Before continuing, let me introduce a terminological simplification. As we see above, a paycheck pronoun itself is understood as an identity function over functions of type $\langle\mathrm{e}, \mathrm{e}\rangle$ ). Technically speaking, then, it doesn't mean anything to talk about its "antecedent" (or to talk about it being "anaphoric" to something else). What happens is simply that the entire clause which contains a "free" paycheck is a function of type $\langle\langle\mathrm{e}, \mathrm{e}\rangle, \mathrm{t}\rangle$ which is then applied to a contextually salient function of type $\langle\mathrm{e}, \mathrm{e}\rangle$. Nonetheless, for expository ease, let me refer to the NP whose meaning supplies the contextually salient function as the "antecedent" to the paycheck pronoun itself, and let me refer to the paycheck pronoun as "anaphoric" to that antecedent. This terminological simplification will do no harm, but will simplify the comparison with the standard account.

The above analysis of why contrastive stress is impossible in (24) makes a striking prediction: which is if the antecedent function can be supplied in some other way - e.g., by the meaning of some other linguistic expression - then contrastive stress will be possible. And this is exactly what happens in the case of the good example (28). ${ }^{5}$ Here the "antecedent" to the second SHE is HIS mother and so this is what supplies the appropriate function, and thus it also contrasts with the first she. The whole picture of how this works is sketched in (37); we repeat the example here with numberings for convenience:

(37) Every man who loves his ${ }_{1}$ mother thinks that she ${ }_{1}$ is nice, while every man who HATES HIS 2 mother thinks that $\mathrm{SHE}_{2}$ is a jerk.

his $_{1}$ : identity function over mother-lovers - hence:

his 1 mother: function from mother-lovers to mothers

hence: appropriate antecedent for she 1

she $_{1}$ : identity function over functions with domain mother-lovers

his 2 : identity function over mother-haters - hence:

his 2 mother: function from mother-haters to mothers hence: appropriate antecedent for $\mathrm{she}_{2}$

$s_{2} e_{2}$ identity function over functions whose domain is mother-haters

Note, then, that she 1 and she 2 contrast.

Why does this work, while the "different variable names solution" doesn't? The difference between the two is crucially tied to one of the main differences between the two accounts of binding. Since in the standard account binding requires some actual variable in the position of "bound" material (rather than binding being a merging of argument slots via the $\mathbf{z}$ rule), it follows that the paycheck pronoun must be complex, as it must contain an actual variable as argument of the paycheck function (or, as part of the lexical material). Thus the paycheck pronoun she or her in the relevant examples is represented as $\mathrm{g}(\mathrm{x})$ or as the mother of $\mathrm{x}$. The problem then is that the function part of the paycheck pronoun and its "antecedent" (the $\underline{f}$ part, or the other linguistic material) can be the same and 
so be in an anaphoric relationship, while the argument variables can contrast. This problem doesn't arise in the variable-free account precisely because in this account there is no actual argument variable of the paycheck function. Rather, there is only an argument slot. This result is that if this function contrasts with some other function, it is because the domains of the two are in contrast - not because the arguments of the two functions contrast. But this in turn means that the paycheck pronoun her in (24) cannot also be anaphoric to his mother (or, to mother).

\section{The standard account revisited: can this solution be mimicked?}

The basic idea in the above account is that the paycheck pronoun cannot both be anaphoric to another NP and at the same time contrast with it. Moreover, this followed in the variable-free account but not in the standard account; the latter floundered because the paycheck pronoun is complex and hence part could be anaphoric while part is in contrast.

Nonetheless, one might wonder if we cannot tweak the standard account in some way as to arrive at an analogous solution. I can think of two obvious ways to try to mimic the story above, but each has problems. Obviously this will not be an exhaustive survey of all ways we could try to account for the facts under the standard account, but hopefully this exercise will give a representative sample of the kinds of problems that the standard account is likely to face.

First, let me restate what we have apparently discovered so far. I am assuming that the reason that contrastive stress is good in (28) but not in (24) is that in the former the paycheck pronoun is not also anaphoric to the material it is contrasting with (it is anaphoric to something else), while in the latter case it is. This fact falls out naturally in the variable-free account because the paycheck pronoun is not complex - it cannot have a piece which is in contrast to something else and another piece which is anaphoric to it. Thus in order to arrive at the same basic generalization in the standard account, we can continue to let the paycheck pronoun be complex but to require full identity between it and its "antecedent". Hence it could not both be anaphoric to some other NP and at the same time be in contrast with it. We will thus consider two ways to flesh out this idea.

Note first, though, that full identity makes sense only under the pronouns of laziness approach, and not under the free function variable approach. The latter specifically claims that it is only the function part which is "seeking" something to supply its value; there should be no reason at all why the individual variable which is the argument of the free function variable should have to be identical to the variable which is the argument of the antecedent. (The pronouns of laziness approach, on the other hand, could be formulated so as to require complete identity of the entire antecedent and the material which is the surface paycheck pronoun, and so an exact identity requirement would not be surprising under this approach.) Thus to the extent that we have already seen arguments against the pronouns of laziness approach, our attempt to mimic the variable-free predictions already has problems. Moreover, we have already seen that full identity is in fact not required; (23), for example, is perfect despite the gender mismatch.

But let us push these problems aside, and move ahead. First we consider a fairly obvious way to try to require full identity. Thus in the discussion of the pronouns of laziness in Sec. 6, we implicitly assumed that the pronominalized material must be identical to its antecedent up to alphabetic variance. That is, the full NP representations of the paycheck pronoun and its antecedent can contain different variable names. Suppose, however, we were to require exact identity, including identity of all variable names. Then we can tell a story which is exactly analogous to the one told above. The representation for (21) must be (38) (I will underline the material which corresponds to the paycheck pronoun): 
every third grade boy $\lambda x$ [x loves $x^{\prime}$ s mother $]$. every fourth grade boy $\lambda x[\mathrm{x}$ loves $\mathrm{x}$ 's mother $]$

The underlined material can surface as a pronoun in virtue of its exact identity to the antecedent (in bold). But then, of course, it cannot contrast, since there is no difference in variable names. Hence no piece of the paycheck pronoun could be in contrast with anything within the antecedent. Moreover, the good case (28) follows just as it did in the variable-free account. Here the underlined material is a paycheck pronoun in virtue of its exact identity with the antecedent (which is indicated in boldface), but here $y$ contrasts with $x$ in the first clause (note that $x$ 's mother in the first clause also corresponds to a surface paycheck pronoun, as it is identical to its boldface antecedent):

every' $\left(\lambda x\left[\operatorname{man}(x) \& x\right.\right.$ loves $x^{\prime}$ 's mother $\left.]\right)(\lambda x[x$ thinks $\underline{x ' s \text { mother }}$ is nice]) while

every' $\left(\lambda y\left[\operatorname{man}(y) \&\right.\right.$ y hates $y^{\prime}$ s mother $\left.]\right)(\lambda y[y$ thinks y's mother is a jerk])

This looks like a straightforward translation of the variable-free account above, and since it relies on fairly natural assumptions it might look like the variable-free account has no particular advantage here (except. of course, for the facts discussed earlier: the pronouns of laziness approach is in any case suspect, and the different variable names approach continues to have the problems discussed in Sec. 3.) But in fact this solution cannot be maintained. Quite strikingly, we cannot actually maintain an exact identity condition on paycheck pronouns. The problem centers on examples like (40):

$$
\begin{aligned}
& \text { Every } \operatorname{man}_{\mathrm{i}} \text { who loves his } \mathrm{s}_{\mathrm{i}} \text { mother thinks that every } \operatorname{man}_{\mathrm{j}} \text { who hates } \\
& \text { her } \left._{\mathrm{f}(\mathrm{j})} \text { should call her } \operatorname{si}_{\mathrm{f}}\right) \operatorname{her}_{\mathrm{f}(\mathrm{j})}
\end{aligned}
$$

This has two readings; the second occurrence of her can be understood in either of the ways shown above. If exact identity were required, a meaning would be lost: the representation for (40) would have to be roughly along the lines of (41):

(41) for every $x$ such that man'( $x)$ and $x$ loves $x$ 's mother: $x$ thinks that for every $x$ such that man'(x) and $x$ hates $\underline{x}$ 's mother $\mathrm{x}$ should call $\underline{\mathrm{x} \text { 's mother }}$

Under the standard ways to interpret variables, the lowest occurrence of $x$ 's mother in this representation (i.e., the lowest paycheck pronoun her) will only be understood under the reading where $\underline{x}$ is bound by the mother-hater. In order to represent the meaning where her is understood as the mother of the mother-lover, we will have to use a different variable name for the mother-hater binder. But then we cannot get the first her to be a paycheck pronoun on the relevant reading.

A second attempt to mimic the variable-free solution would be to use sorted variables, where we have a different set of variables for each domain. Suppose then that we allow two variables to contrast not in virtue of the fact that they have different names, but in virtue of the fact that they are variables over different domains. (Note that this avoids the problems discussed in Sec. 3.) Suppose further that a paycheck pronoun must be identical to its antecedent up to alphabetic variance (the variable names can differ), but that any variables within the antecedent and the pronoun must be variables over the same domain. Space precludes showing the 
details, but the interested reader can verify that this will yield the same result as the variable-free solution. However, it requires an infinite number of types of variable names (as well as an infinite number of variables themselves) since there are presumably an infinite number of possible domains that we must sort over. This seems to me to be a rather unpleasant result of having variables, although I leave it to the variable aficionados to decide whether this is a result that can be lived with. ${ }^{7}$ And of course it continues to require the pronouns of laziness analysis.

\section{Unsolved problems and open questions}

We leave this discussion with two open questions/unsolved problems. First, it will ultimately be necessary to spell out just what kind of functions come with contextually specified domain restrictions. To give the most obvious example of a potential problem, consider the impossibility of contrastive stress in (42):

$$
\text { *Every third grade boy ran, and every FOURTH grade boy RAN. }
$$

But one can imagine a story to be told if this were good: run denotes a function of type $\langle\mathrm{e}, \mathrm{t}\rangle$ but where the domain of the function is contextually specified. In (42), the two occurrences of ran have different domains supplied. We need a principled explanation for why this is not possible in (42), but why pronouns can contrast on the basis of the fact that they can be functions over different contextually supplied domains.

A second unsolved problem is that a version of (28) seems possible even if there is no stress on the second his:

(43) Every $\operatorname{man}_{i}$ who loves his $s_{i}$ mother thinks that she $e_{f(i)}$ is nice, while every

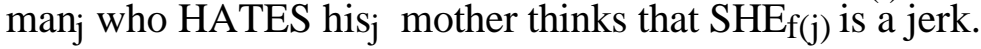

I have checked this with several informants, and most felt that (43) was not as good as (28), where the second HIS is stressed. However, all - including myself - find (43) to be not terribly bad. This is surprising. Since it is his 2 mother which supplies the antecedent function for the stressed $S H E_{2}$, it must be the case that his here is understood as the identity function over mother-haters while the first his is understood as the identity function over mother-lovers. These two should thus contrast, and so the second his should require stress.

\section{Endnotes}

* This research was supported by NSF Grant SBR \# 98-50552.

${ }^{1}$ I will consider here only to consider a portion of Sauerland's arguments; he deals not only with cases where contrastive stress is allowed but also with a case where it is required, and attempts to use this as an argument for variables. Space precludes discussion of this; suffice it to say that the crucial judgments are at best delicate.

2 The idea would be to treat stress as a feature, and to build the agreement in to the $\mathbf{z}$ rule. Thus (4) gives only the semantics of $\mathbf{z}$, but in Jacobson (1999, to appear) I have hooked this in to a syntax, where the entire program is implemented in a Categorial Grammar syntax. I skip the details here, but the basic idea would be to have the syntactic portion of the $\mathbf{z}$ rule require agreement of the stress feature both on the binder position and on the superscript feature which records the existence of a pronoun.

3 One might, however, wonder why it is relatively difficult to get a paycheck reading without an overt antecedent. My hunch is that functions of type $\langle\mathrm{e}, \mathrm{e}\rangle-$ 
unlike ordinary individuals - are difficult to make contextually salient, and hence are easiest to access if made salient by the meaning of overt linguistic material.

4 The idea that contrastive stress is allowed only if the "head" - or, function - part of the paycheck pronoun contrasts with something else has the advantage of correctly predicting that contrastive stress is allowed in (i):

Every boy who loves his mother and hates his father thinks that SHE is nice and that HE is horrible.

Here the argument variables in $S H E$ and in $H E$ are the same, but the "heads" or function parts are different.

However, it should be noted that an appeal to the notion "head" is actually quite problematic under the free function variable approach. As noted in the text, this would force us to think of the function variable as the head, and the individual argument variable as its complement. But in fact this is not tenable. The reason is that, as detailed in Engdahl (1986), the function variable can actually be a variable over n-place functions applied to $\mathrm{n}$ individual argument variables. For example, we have paycheck cases like (ii), and even more complex cases can be constructed:

(ii) The woman $_{i}$ who told Sears $j$ that the money she ${ }_{i}$ owed them $_{j}$ was in the mail was wiser than the woman $_{k}$ who told Filene's 1 that $\mathrm{it}_{\mathrm{f}(\mathrm{k}, \mathrm{l})}$ had not yet been mailed.

It thus makes little sense to see this as analogous to a head/complement situation. (I thank Gennaro Chierchia for this observation.)

5 This is also what happens in the case of (i) in fn. 4.

6 Because of the problems noted in Sec. 3, Sauerland has also abandoned the "different variable names" solution, and proposes an alternative within the standard theory which is closer to the alternative here (Sauerland, this volume). This is to allow bound pronouns to be represented by copying the lexical material from the heads of their antecedents. (Hence if the heads of the antecedents can contrast the bound pronouns can also contrast.) Note, though, that examples like (28) are potentially problematic for this view. The reason concerns stress on HIS within the second relative clause. The heads of both NPs are the same (both are man); the contrast comes only by considering the entire relative clause. However, if the representation of a stressed pronoun involves copying its entire NP antecedent, then we revisit the infinite regress problem which led to the abandonment of a pronominalization transformation some 30 years ago. (Thus the "antecedent" for his is the entire NP in which his is contained.) Possibly this could be solved by allowing some sort of copying here of a portion of the relative clause, but the details of just what gets copied would need to be spelled out.

\section{References}

Cooper, R. (1979). "The Interpretation of Pronouns", in F. Heny and H. Schnelle (eds.), Syntax and Semantics 10: Selections from the Third Groningen Round Table, 61-92. Academic Press, New York.

Dowty, D. (1999). "Natural Language Anaphora and Type Logical Syntax", paper presented at the Formal Grammar 1999 conference, Utrecht.

Engdahl, E. (1986). Constituent Questions. Reidel, Dordrecht. 
Geach, P. (1972). "A Program for Syntax", in D. Davidson and G. Harman (eds.), Semantics of Natural Language, 483-497. Reidel, Dordrecht.

Hepple, M. (1990). The Grammar of Order and Dependency: A Categorial Approach. Ph.D. Dissertation, University of Edinburgh.

Heim, I. (1990). "E-type Pronouns and Donkey Anaphora", Linguistics and Philosophy 13, 137-178.

Jacobson, P. (1977). The Syntax of Crossing Coreference Sentences. Ph.D. Dissertation, University of California at Berkeley. Published by Garland Press, 1980, New York.

Jacobson, P. (1991). "Bach-Peters Sentences in a Variable-Free Semantics", in P. Dekker and M. Stokhof (eds.), Proceedings of the Eighth Amsterdam Colloquium. University of Amsterdam ILLC, Amsterdam.

Jacobson, P. (1999). "Towards a Variable-Free Semantics", Linguistics and Philosophy 22, 117-184.

Jacobson, P. (to appear). "Paycheck Pronouns, Bach-Peters Sentences, and Variable Free Semantics", Natural Language Semantics.

Karttunen, L. (1969). "Pronouns and Variables" in R. Binnick et al. (eds.), Papers from the Fifth Regional Meeting of the Chicago Linguistic Society, 108116. Chicago Linguistic Society, Chicago.

Montague, R. (1974). "The Proper Treatment of Quantification in Ordinary English", in R. Thomason (ed.), Formal Philosophy: Selected Papers of Richard Montague, 247-278. Yale University Press, New Haven.

Partee, B. (1975). "Deletion and Variable Binding", in E. Keenan (ed.), Formal Semantics of Natural Language. Cambridge University Press, Cambridge.

Rooth, M. (1985). Association with Focus. Ph.D. Dissertation, University of Massachusetts, Amherst.

Ross, J. (1967). Constraints on Variables in Syntax. Ph.D. Dissertation, MIT, Cambridge.

Sauerland, U. (1998). The Meaning of Chains. Ph.D. Dissertation, MIT, Cambridge.

Sauerland, U. (1999). "Why Variables?", in Proceedings of the North East Linguistic Society 29, 323-337. Distributed by University of Massachusetts GLSA, Amherst.

Sauerland, U. (this volume). "On the Content of Pronouns: Evidence from Focus". Proceedings of SALT 10, Ithaca, Cornell Working Papers in Linguistics.

Schwarzschild, R. (1999). "GIVENness, AVOIDF and Other Constraints on the Placement of Accent", Natural Language Semantics 7, 141-177. 
Szabolcsi, A. (1992). "Combinatory Categorial Grammar and Projection from the Lexicon", in I. Sag and A. Szabolcsi (eds.), Lexical Matters. CSLI Publications, Stanford. 